\title{
Report
}

\section{A Japanese Woman with Stevens-Johnson Syndrome without Skin Lesions Induced by an Initial Dosage of Lamotrigine}

\author{
Yasuyuki Sumida ${ }^{1,}{ }^{*}$, Hidetaka Ishino ${ }^{2}$, Atsushi Saitou ${ }^{3}$, Yohei Otsuki ${ }^{4}$, Satoshi Komori ${ }^{5}$, \\ Atsuhiro Kanzaki ${ }^{6}$ \\ ${ }^{1}$ Emergency Department, North Medical Center Kyoto Prefectural University of Medicine, Yosanocho, Japan \\ ${ }^{2}$ Department of General Internal Medicine, North Medical Center Kyoto Prefectural University of Medicine, Yosanocho, Japan \\ ${ }^{3}$ Department of Otolaryngology, Kyoto Second Red Cross Hospital, Kyoto, Japan \\ ${ }^{4}$ Department of Ophthalmology, Kyoto Prefectural University of Medicine, Kyoto, Japan \\ ${ }^{5}$ Department of Dermatology, Kyoto Prefectural University of Medicine, Kyoto, Japan \\ ${ }^{6}$ Department of Psychiatry, Kyoto Prefectural University of Medicine, Kyoto, Japan
}

Email address:

y-sumida@koto.kpu-m.ac.jp (Y. Sumida)

${ }^{*}$ Corresponding author

\section{To cite this article:}

Yasuyuki Sumida, Hidetaka Ishino, Atsushi Saitou, Yohei Otsuki, Satoshi Komori, Atsuhiro Kanzaki. A Japanese Woman with Stevens-Johnson Syndrome without Skin Lesions Induced by an Initial Dosage of Lamotrigine. American Journal of Clinical and Experimental Medicine. Vol. 7, No. 1, 2019, pp. 7-11. doi: 10.11648/j.ajcem.20190701.12

Received: December 16, 2018; Accepted: February 27, 2019; Published: March 19, 2019

\begin{abstract}
Atypical Stevens-Johnson syndrome (SJS) which is characterized by little or no skin involment, is reported to be triggered by less medications than infection. There are few reports with atypical SJS induced by medications. This is a case report of atypical SJS induced by Lamotrigine (LTG). A 31-year-old Japanese woman, who was bipolar disorder and prescribed an initial dosage of LTG $25 \mathrm{mg} /$ day ten days ago, presented with high fever and throat pain for two days. At first she was suspected of pharyngitis and administered antibiotics. She was hospitalized on the first visit day, but she was getting worse and fell into shock over several days. Furthermore she presented with erosions and bleeding of her lips and oral mucosa on day6, and so she was diagnosed with SJS, but her skin lesions were mild even at the extreme of the clinical course. She gradually developed acute pulmonary failure, liver dysfunction, coma and Disseminated Intravascular Coagulation (DIC). But she was treated with intensive care consisting of steroid pulse followed by high dose corticosteroids, and so she gradually recovered from SJS and corticosteroids could be tapered. At last she was discharged on day50 and had no sequelae with SJS. It was extremely difficult to diagnose this case as atypical SJS because of the unremarkable skin lesions and similarity to pharyngitis. It was also characteristic that she was already in severe status when it satisfied the diagnostic criteria for SJS. But physicians should not miss from the perspective of the fatality and social problem. It should be noted that atypical SJS shows mild or little cutaneous lesions.
\end{abstract}

Keywords: Atypical, Incomplete, Fuchs Syndrome, Lamotrigine, Stevens-Johnson Syndrome, Without Skin Lesions, Without Skin Involvement

\section{Introduction}

Stevens-Johnson syndrome (SJS) is characterized by severe mucocutaneous reactions, triggered by medications and infections. There are some reports with atypical presentation of SJS which is characterized by muco-membranous lesions and little or no skin lesions. Because of its feature it is reported to be challenging to diagnose atypical SJS. This is a case report of a Japanese woman with atypical SJS induced by Lamotrigine (LTG) which was extremely difficult to diagnose. 


\section{Case Report}

A-31-year-old Japanese woman, who was diagnosed with bipolar disorder four months ago, was prescribed LTG $25 \mathrm{mg}$ /day ten days ago. She had been under medications lithium carbonate $800 \mathrm{mg}$, flunitrazepam $1 \mathrm{mg}$ and eszopiclone $2 \mathrm{mg}$ until then. She had suffered from fever $38.0^{\circ} \mathrm{C}$ for two days, and she was brought by his mother. She presented with high fever $40.0^{\circ} \mathrm{C}$ and throat pain without cough and sputum. Initial vital signs were: pulse $97 / \mathrm{min}$; blood pressure $155 / 84 \mathrm{mmHg}$; respiratory rate $24 / \mathrm{min}$; and saturation of peripheral oxygen $97 \%$ on room air. She had bilateral eye lid edema, mild conjunctivitis and red pharynx at first visit. She had no skin lesions and no allergies to medications.

Laboratory examinations revealed white blood cell count $6000 / \mathrm{mm} 3$, (neutrophilia 90.6\%), hemoglobin level $12.8 \mathrm{~g} / \mathrm{dl}$, lower platelets count $85000 / \mathrm{mm} 3$, mild coagulopathy (prothrombin time 13.3sec (normal range $=10.8-12.8 \mathrm{sec}$ ), fibrin degradation product $72.0 \mathrm{ug} / \mathrm{ml}$ (normal range = $0.0-10.0 \mathrm{ug} / \mathrm{ml})$ ), mild elevated levels of C-reactive protein $5.2 \mathrm{mg} / \mathrm{dl}$, mild liver dysfunction (AST $71 \mathrm{IU} / \mathrm{L}, \mathrm{LDH}$ 481IU/L), normal CPK and kidney function. Urine analysis was normal.

She felt general weakness due to high fever and so she was hospitalized on the first visit day. At first, she was suspected of bacterial pharyngitis and administered antibiotics (ceftriaxone $2 \mathrm{~g}$ /day), but she gradually fell into shock and was moved to the intensive care unit (ICU) on day3 (Figure 2). All drugs including LTG were discontinued. Bacterial cultures (pharynx, blood, urine) were all negative. Serum level of LTG was $0.91 \mathrm{ug} / \mathrm{ml}$ (normal range $=2.5-15 \mathrm{ug} / \mathrm{ml}$ on 3rd day). She got unstable psychiatrically, and so she had an urge to take LTG again. As a result of consult with the psychiatrist, she was allowed to take LTG $25 \mathrm{mg}$ on day4. That turned out to be drug challenge test, that is, she presented with generalized maculopapular eruptions (Figure 3) and body temperature rose up to $40^{\circ} \mathrm{C}$ again. According to above clinical course she was diagnosed with drug eruption induced by LTG. She was suspected of erythema multiforme major so that intravenous prednisolone (PSL) 30mg/day was started. But she presented with erosions and bleeding of her lips and oral mucosa on day6 (Figure 4), and so she was diagnosed as SJS satisfying all three major criteria of diagnostic criteria for SJS (Figure 5) [1]. However she had mild erosions of the cornea and no genital mucosal lesions. Steroid pulse (methyl-prednisolone (mPSL) 1g/day for three days) followed by high dose corticosteroid administration (PSL 60mg/day) was started. She gradually developed acute pulmonary failure (due to pleural effusion), liver dysfunction, coma and Disseminated Intravascular Coagulation (DIC). She temporarily required non-invasive positive pressure ventilation (NPPV) for three days, but she was gradually getting better and discharged from ICU on day18 and her oral mucosa progressively resolved and showed re-epithelization. Corticosteroids could be tapered, and she was discharged on day50 with PSL $7.5 \mathrm{mg}$ /day. PSL was stopped on day90 at outpatient visit. She had no sequelae associated with SJS.
Drug-induced lymphocyte stimulation test (DLST) for LTG on day22 was positive.

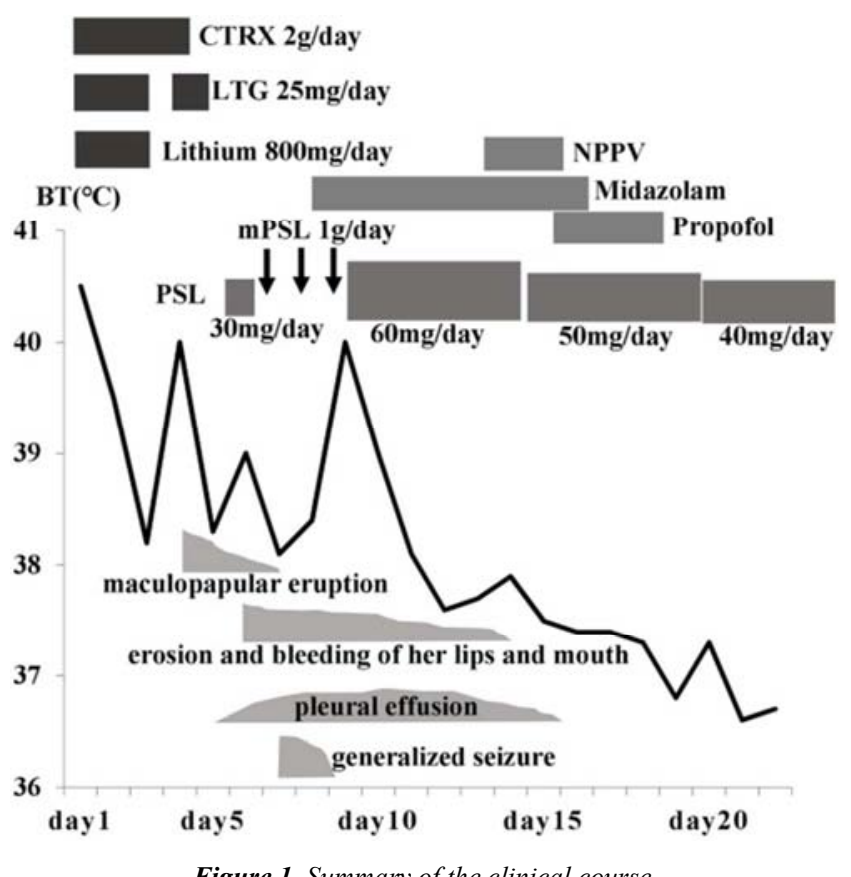

She presented with throat pain and fever on hospitalization (day1), but gradually she was getting into shock. She was treated in the ICU (intensive care unit) between day3 and day18. On day4 she presented with maculopapular eruption of her trunk and extremities soon after re-exposure to LTG $25 \mathrm{mg}$. On day7 she presented with erosions and bleeding of her lips and oral cavity. So we diagnosed SJS due to LTG and treated with steroid pulse (mPSL 1g/day x 3days) followed by intravenous PSL $60 \mathrm{mg} /$ day. PSL was gradually decreased every five days. She suffered from generalized seizure during day7-9 and pleural effusions during day5-16, but she was treated with anticonvulsants and NPPV, and then she was gradually getting better and discharged ICU on day18.

$\mathrm{Y}$ axis means body temperature (BT).

CTRX: ceftriaxone, LTG: lamotrigine, PSL: prednisolone, mPSL: methyl-prednisolone, NPPV: non-invasive positive pressure ventilation facial edema, especially bilateral eyelids` edema on day 3 .

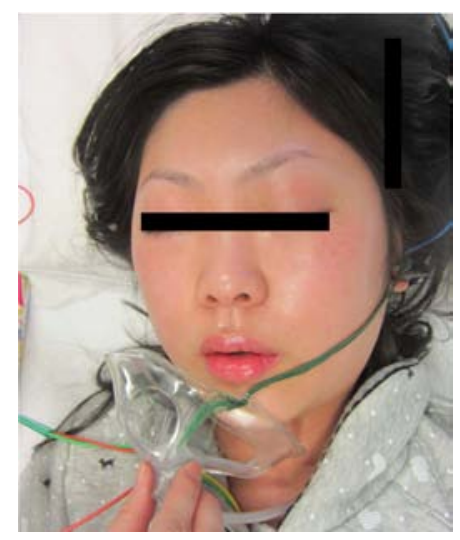

Figure 2. Facial edema (day3). 


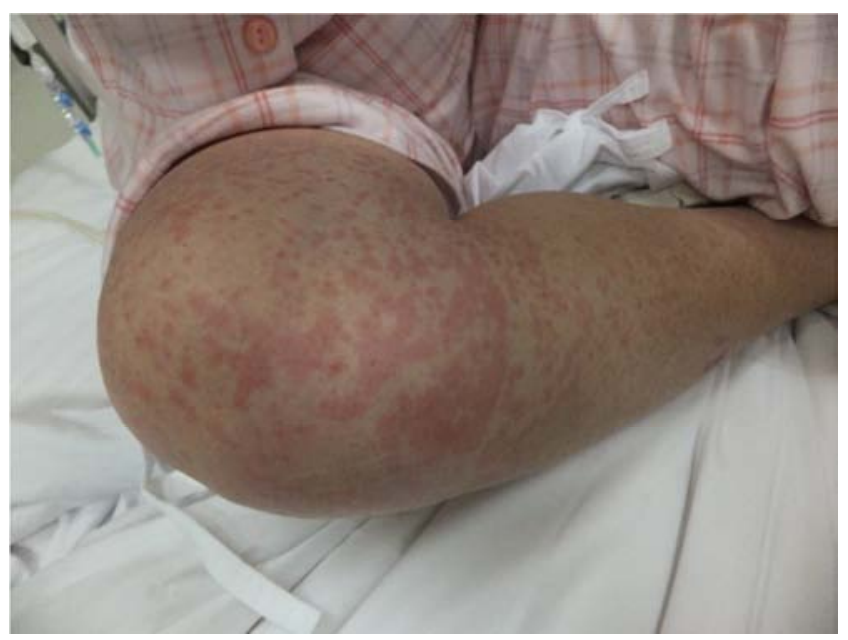

Figure 3. Maculopapular eruption of her knee (day4).

maculopapular eruption of her trunk and extremities after re-exposure to LTG $25 \mathrm{mg}$ on day4.

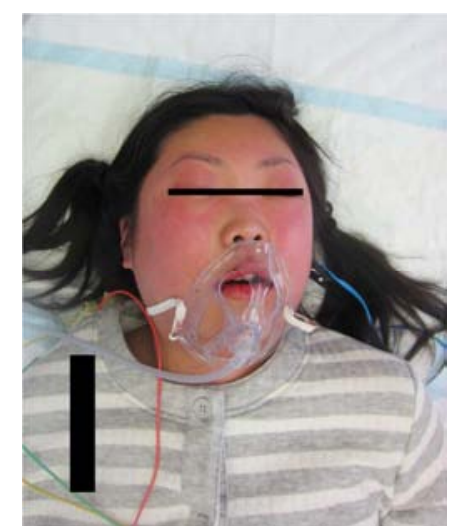

Figure 4. Erosions and bleeding of her lips (day7).

She presented with general edema. Furthermore we found erosions and bleeding of her lips and oral mucosa. But on this photograph we can slightly see erosions and bleeding of her lips. According to thease symptoms, we clinically diagnosed SJS due to LTG on day7.

\section{Diagnostic criteria for Stevens-Johnson syndrome}

\section{(1) Introduction}

Stevens-Johnson syndrome is severe mucocutaneous eruption with fever of such as lips, conjunctivas, genital areas et al, and accompanies with epidermal necrosis such as bullae and detachment of the epidermis, commonly triggered by medications.

(2) Major criteria (mandatory)

Severe mucocutaneous eruptions hemorrhagic or congestive

Skin lesion (erosion or bullae) is $<10$ percent of the body surface

Fever higher than $38^{\circ} \mathrm{C}$

\section{(3) Minor criteria}

Atypical target erythema multiforme

Bilateral nonspecific conjunctivitis with corneal abrasion and/or pseudomembrane

Histopathologic epidermal necrosis

* However, Stevens-Johnson syndrome can deteriorate into toxic epidermal necrolysis, and so must be reevaluated at the advanced stage.

All three major criteria are required to diagnose Stevens·Johnson syndrome.

Figure 5. Japanese diagnostic criteria for Stevens-Johnson syndrome [1].

\section{Discussion}

At last we managed to diagnose SJS due to LTG based on severe erosions and bleeding of oral mucosa, but it was difficult to diagnose because a number of other diseases including autoimmune diseases and infections can manifest with fever and mucosal change. Even at the advanced stage she was found only oral erosions and bleeding. Her skin lesions and conjunctivitis were mild. Some cases like ours have been reported as "incomplete/atypical/variant Stevens-Johnson syndrome”, "Stevens-Johnson syndrome without skin lesions/involvement" and "Fuchs syndrome (FHS)". It is considered a rare variant SJS which is characterized by muco-membranous lesions and little or no skin involvement [2-7]. Most of atypical SJS are triggered by infections; Herpes simplex virus (HSV) and Mycoplasma pneumoniae (M.pneumoniae) are common. There are more reports with atypical SJS by M.pneumoniae, especially in children and young people [5-13]. But there are few reports of atypical SJS triggered by medications. Some physicians reported atypical SJS induced by M.pneumoniae as M.pneumoniae-induced rashes and mucositis (MIRM) or 
M.pneumoniae-associated mucositis (MPAM), from the view point of separate entity $[12,13]$. Theresa N. Canavan et al reviewed characteristics of 202 cases (95articles) with MIRM. Cutaneous involvement ranged from absent (34\%), to sparse (47\%), to moderate (19\%). Oral, ocular, and urogenital mucositis was reported in $94 \%, 82 \%$, and $63 \%$ of cases respectively [12]. That is to say, MIRM tends to be more frequent with muco-membranous lesions than cutaneous involvement. The majority of patients (81\%) made a full recovery. MIRM is reported to have a milder clinical course with rare long-term sequelae and mortality [12]. This case temporarily fell into severe status, and the clinical course was not mild. It might be because triggered by drug. David A. Wetter reported likewise that M. pneumoniae induced SJS was less severe than drug-induced SJS [14]. Moreover it was characteristic that this case showed only severe oral mucositis though triggered by drug. Patients with SJS induced by $M$. pneumoniae seem to show ocular manifestations more frequently than those with drug-induced SJS. On the other hand, oral mucositis is surely caused in both types, and the frequency seems to be no difference $[13,15]$.

This case has a limitation that the serology and PCR of $M$. pneumoniae and HSV were not checked, but it's sure that SJS was induced by LTG according to DLST and the clinical course (in particular on day4 accidental drug challenge test by re-taking LTG).

LTG is an anticonvulsant drug for epilepsy and a mood-stabilizing drug for bipolar disorder. The most concerning of this adverse reactions is SJS/toxic epidermal necrolysis (TEN). The incidence of SJS/TEN is $0.04 \%$, which is the highest frequency in anti-epileptic drugs [16]. But LTG is less teratogenic so that there is a tendency to be prescribed for young women. Moreover it is expected that the prescription frequency will not decrease in future because it is one of the few drugs effective in preventing recurrence of depressive episodes of bipolar disorder.

Making the diagnosis of atypical SJS is clinically challenging, especially because the skin may be completely unaffected. It can be an under-recognized diagnosis [2]. This case got into shock in a few days before manifesting mucosal lesions enough for satisfying the diagnostic criteria for SJS, so that the diagnosis and therapy was delayed. It was difficult to diagnose earlier before severe status even if looking back on it later.

\section{Conclusion}

This case was atypical SJS induced by LTG which was extremely difficult to diagnose. We alert clinicians that atypical SJS may be induced by drugs and may not be mild. It is challenging to diagnose atypical SJS because of little or no skin lesions. But physicians should not miss from the perspective of the fatality and social problem.

This report is based on a study first reported in [Yasuyuki Sumida et al: A case of 31-year-old woman with bipolar disorder presented with Stevens-Johnson syndrome induced by an initial dosage of Lamotrigine and ataxic dysarthria possible to be caused by lithium toxicity. Journal of Japanese
Association for Acute Medicine 2016; 27:270-6].”

\section{Informed Consent}

Yes.

\section{Registry and the Registration}

None.

\section{Conflict of Interest}

None

\section{References}

[1] Michiko Aihara, Yoko Kano and Masahumi Iijima et al (2009). Guidelines for the Management of Stevens-Johnson Syndrome and Toxic Epidermal Necrolysis 2009, Established by the Japanese Research Committee on Severe Adverse Reaction (J-SCAR) Supported by the Ministry of Health, Labour and Welfare of Japan. Japanese Journal of Dermatology 119, 2157-63 (in Japanese).

[2] Havliza K, Jakob A and Rompel R (2009). Erythema multiforme majus (Fuchs syndrome) associated with mycoplasma pneumonia (Stevens-Johnson syndrome without skin lesions). J Dtsch Dermatol Ges 7, 445-7.

[3] Meyer Sauteur PM, Gansser-Kälin U and Lautenschlager S et al (2011). Fuchs syndrome associated with mycoplasma pneumoniae (Stevens-Johnson syndrome without skin lesions) Pediatr Dermatol 28, 474-6.

[4] Thomas M. Nappe, Stephanie L. Goren-Garcia, and Jeanne L. Jacoby (2016). Stevens-Johnson syndrome after treatment with azithromycin: an uncommon culprit. Am J Emerg Med 34, 676.e1-3.

[5] Shailendra Kapoor (2016). Fuchs syndrome: A rare and unique variant of Stevens-Johnson Syndrome. Am J Emerg Med 34, 910.

[6] Kayi Li and Richard M. Haber (2012). Stevens-Johnson syndrome without skin lesions (Fuchs syndrome): a literature review of adult cases with mycoplasma cause. Arch Dermatol $148,963-4$.

[7] Patrick M. Meyer Sauteur, Philippe Goetschel and Stephan Lautenschlager (2012). Mycoplasma pneumoniae and mucositis-part of the Stevens-Johnson syndrome spectrum. J Dtsch Dermatol Ges 10, 740-6.

[8] Peter C. Schalock, and James G. H. Dinulos (2005). Mycoplasma pneumoniae-induced Stevens-Johnson syndrome without skin lesions: fact or fiction?. J Am Acad Dermatol 52, 312-5.

[9] Rémy Gossart, Eve Malthiery, Fanny Aguilar, Jacques-Henri Torres, and Marie-Alix Fauroux (2017). Fuchs Syndrome: Medical Treatment of 1 Case and Literature Review. Case Rep Dermatol 9, 114-20.

[10] Chun Yi Ting and Raveen Shahdadpuri (2018). Fuchs syndrome: a case of fever, mucositis and conjunctivitis. BMJ Case Rep 2018 Jan 29. 
[11] Reyhan Amode, Saskia Ingen-Housz-Oro, and Nicolas Ortonne et al (2018). Clinical and histologic features of Mycoplasma pneumoniae-related erythema multiforme: A single-center series of 33 cases compared with 100 cases induced by other causes. J Am Acad Dermatol 79, 110-7.

[12] Canavan TN, Mathes EF, and Frieden I et al (2015). Mycoplasma pneumoniae-induced rash and mucositis as a syndrome distinct from Stevens-Johnson syndrome and erythema multiforme: A systematic review. J Am Acad Dermatol 72, 239-45.

[13] I. Vujic, A. Shroff and M. Grzelka et al (2015). Mycoplasma pneumoniae-associated mucositis-case report and systematic review of literature. J Eur Acad Dermatol Venereol 29, 595-8.
[14] David A. Wetter and Michael J. Camilleri (2009). Clinical, Etiologic, and Histopathologic Features of Stevens-Johnson Syndrome During an 8-Year Period at Mayo Clinic. Mayo Clin Proc 85, 131-138.

[15] Kunimi Y, Hirata Y, and Aihara M et al (2011). Statistical Analysis of Stevens-Johnson Syndrome Caused by Mycoplasma pneumonia Infection in Japan. Allergol Int 60, 525-32.

[16] Romi Bloom and Kyle T. Amber (2017). Identifying the incidence of rash, Stevens-Johnson syndrome and toxic epidermal necrolysis in patients taking lamotrigine: a systematic review of 122 randomized controlled trials. An Bras Dermatol 92, 139-41. 